\section{Anemia e deficiência de ferro em pré-escolares da Amazônia Ocidental brasileira: prevalência e fatores associados}

\author{
Anemia and iron deficiency among schoolchildren \\ in the Western Brazilian Amazon: prevalence and \\ associated factors
}

\author{
1 Escola de Enfermagem, \\ Universidade Federal de \\ Minas Gerais, Belo Horizonte, \\ Brasil. \\ 2 Centro de Ciências \\ da Saúde e Desporto \\ Universidade Federal do Acre, \\ Rio Branco, Brasil. \\ 3 Faculdade de Saúde \\ Pública, Universidade de São \\ Paulo, São Paulo, Brasil. \\ Correspondência \\ T. G. Castro \\ Departamento de Enfermagem \\ Materno-Infantil e \\ Saúde Pública, Escola de \\ Enfermagem, Universidade \\ Federal de Minas Gerais. \\ Av. Alfredo Balena 190, \\ Belo Horizonte, $M G$ \\ 30130-100, Brasil. \\ tgontijo108@gmail.com
}

\begin{abstract}
This cross-sectional population-based study investigated prevalence rates and associated factors for anemia, iron deficiency anemia, and iron deficiency among children 6 to 60 months of age in two towns in Acre State, Brazil $(N=624)$. Hemoglobin, plasma ferritin, and soluble transferrin receptor were measured in venous blood samples. Socioeconomic, demographic, and disease data were obtained using a questionnaire. Prevalence ratios were calculated by Poisson regression in a hierarchical model. Prevalence rates for anemia, iron deficiency anemia, and iron deficiency were $30.6 \%, 20.9 \%$, and $43.5 \%$, respectively. Children younger than 24 months showed higher risk of anemia, iron deficiency anemia, and iron deficiency. The highest family income tertile was a protective factor against iron deficiency anemia (PR: 0.62; 95\%CI: 0.40-0.98). The highest heightfor-age quartile was protective against anemia (0.62; 0.44-0.86) and iron deficiency anemia ( 0.51 ; 0.33-0.79), and recent history of diarrhea was associated with increased risk of anemia (1.47; 1.12-1.92) and iron deficiency anemia (1.44; 1.032.01). Geohelminth infection was associated with increased risk of anemia, iron deficiency anemia, and iron deficiency.
\end{abstract}

Anemia; Iron Deficiency; Child Welfare
Teresa Gontijo de Castro 1

Mônica Silva-Nunes 2

Wolney Lisboa Conde 3

Pascoal Torres Muniz 2

Marly Augusto Cardoso 3

\section{Introdução}

A Organização Mundial da Saúde (OMS) define anemia como o estado onde a concentração de hemoglobina está anormalmente baixa como conseqüência da deficiência de um ou mais nutrientes essenciais, qualquer que seja a origem da carência 1. A deficiência de ferro é apontada como a determinante causal principal da maioria dos casos de anemia, e é, atualmente, a carência nutricional mais prevalente no mundo, afetando países desenvolvidos e em desenvolvimento 1 . Em 2002, a anemia ferropriva foi considerada um dos mais importantes fatores contribuintes para a carga global de doenças 2. Dentre as populações em risco para a anemia ferropriva, as crianças em idade pré-escolar constituem grupo vulnerável, suscitando preocupação pelos prejuízos que acarreta: comprometimento do desenvolvimento mental, retardo de crescimento e desenvolvimento físico, aumento na freqüência de morbidades, dentre outros ${ }^{1}$.

Pesquisa nacional estimou prevalência de $20,9 \%$ de anemia entre as crianças menores de cinco anos 3. Outros estudos nacionais localizados apontam prevalências de anemia que oscilam de $36,4 \%$ a $47,8 \%$ entre menores de cinco anos $4,5,6,7$ e de $47,8 \%$ a $54 \%$ entre menores de três anos 8,9. Além disso, contrariamente à tendência de redução da desnutrição infantil nas últimas décadas, a anemia parece apresentar 
tendência temporal de aumento entre as crianças brasileiras 10 .

A grande maioria dos estudos populacionais brasileiros disponíveis avaliou prevalências de anemia pela medição única da hemoglobina sanguínea. A utilização isolada desse parâmetro não é suficientemente sensível ou específica para o diagnóstico de estado nutricional de ferro 11 . Sua baixa sensibilidade é explicada pelo fato de que grande proporção de ferro corporal total deve ser depletada antes da ocorrência de alterações nas concentrações de hemoglobina sanguínea. Sua baixa especificidade advém de outras causas de anemia, como outras deficiências nutricionais, infecções, deficiência da desidrogenase glicose- 6 -fosfato e hemoglobinopatias 12 .

Além da necessidade de estudos de base populacional sobre estado nutricional de ferro na infância, é importante a investigação de seus principais determinantes, buscando-se identificar fatores para definição de ações estratégicas de políticas públicas para a promoção da saúde na infância 2,5,6,7,8. O presente estudo investigou prevalências de anemia e fatores associados à anemia, anemia ferropriva e deficiência de ferro entre pré-escolares de 6 a 60 meses residentes em dois municípios da Amazônia Brasileira.

\section{Materiais e métodos}

Tratou-se de estudo transversal de base populacional conduzido com crianças de 6 a 60 meses residentes na área urbana dos municípios de Acrelândia e Assis Brasil, Estado do Acre, Brasil. A coleta de dados foi realizada em janeiro de 2003. Segundo censo dos registros do Programa Saúde da Família (PSF) de dezembro de 2002, com cobertura integral em ambos os municípios, havia 491 domicílios em Acrelândia e Assis Brasil, com o total de 673 crianças de 6 a 60 meses. Foram realizadas entrevistas com questionários estruturados, exames antropométricos e exames laboratoriais (sangue e fezes). A equipe de campo foi constituída por agentes comunitários de saúde, estudantes do curso de Enfermagem da Universidade Federal do Acre (UFAC) e pós-graduandos da Universidade de São Paulo (USP), com treinamento e supervisão local pela equipe de pesquisadores do projeto. Durante as visitas domiciliares os entrevistadores identificaram-se, explicaram os objetivos e benefícios da pesquisa e solicitaram a assinatura de termo de consentimento livre e esclarecido aos pais ou responsáveis pelas crianças, assegurando-lhes o sigilo das informações. O estudo foi aprovado pelo Comitê de Ética em Pesquisa com Seres Humanos da Faculdade de Saúde Pública da USP (protocolo de pesquisa no. 810). As crianças diagnosticadas com infecção parasitária e anemia neste estudo receberam tratamentos medicamentosos adequados, prescritos pela equipe médica do projeto.

Informações sobre a situação demográfica, sócio-econômica, ambiental, antecedentes maternos de história reprodutiva, características ao nascimento, aleitamento materno e morbidades pregressas foram obtidas por meio de questionário estruturado, aplicado aos pais ou responsáveis pelas crianças em entrevistas domiciliares. A diarréia foi definida como a passagem ou perda freqüente de fezes líquidas nos últimos 15 dias anteriores à entrevista. Para avaliação do nível sócio-econômico das famílias, foi calculado um índice de riqueza (em escores z) com base na presença de bens de consumo e utilidades domésticas nos domicílios (televisão, aparelho de som, videocassete, fogão, geladeira, rádio, telefone, bicicleta, ferro elétrico, liquidificador, carro, sofá, máquina de lavar e antena parabólica), utilizando-se da análise de componentes principais, conforme descrição detalhada em publicação anterior 13. Os escores foram somados, estimando-se o índice de riqueza por domicílio, categorizado e analisado em tercis.

As crianças com dois anos ou mais tiveram o peso mensurado pela utilização de balança portátil digital eletrônica (Plenna, Estados Unidos), com capacidade de $150 \mathrm{~kg}$ e precisão de $100 \mathrm{~g}$. A estatura foi medida em estadiômetro com precisão de $0,1 \mathrm{~cm}$. Os menores de dois anos tiveram o peso e o comprimento mensurados com auxílio de balança pediátrica digital, com capacidade de $16 \mathrm{~kg}$ e precisão de $10 \mathrm{~g}$ (Soehnle, Alemanha) e de antropômetro infantil (precisão de $0,1 \mathrm{~cm}$ ), respectivamente. A obtenção das medidas antropométricas seguiu as técnicas da OMS 14. Os índices peso/idade $(\mathrm{P} / \mathrm{I})$, peso/estatura $(\mathrm{P} / \mathrm{E})$ e estatura/idade (E/I) foram calculados em escores z, com auxílio do programa WHO Anthro2005 (OMS; http://www.who.int/childgrowth/softwa re/en/), que se baseia na população de referência da OMS 15. Foram estabelecidos os pontos de corte menor que -2 escore $\mathrm{z}$ para classificação de déficit nutricional para os três índices e maior que +2 escore z para a classificação de excesso de peso com base no índice P/E.

A presença de helmintos e protozoários foi investigada com uso de metodologia descrita por Hoffman et al. 16, mediante uma amostra fecal. Nesta análise, foram considerados geohelmintos os seguintes parasitas intestinais: Ancylostoma duodenale, Ascaris lumbricoides, Strongyloides stercoralis e Trichuris trichura. As concentrações de hemoglobina sanguínea ( $\mathrm{Hb})$, ferritina e receptor de transferrina plasmáticos foram avaliadas pelas amostras de sangue venoso, colhidas 
após jejum de 10 a 12 horas. A mensuração da concentração de hemoglobina foi feita com uso do hemoglobinômetro portátil Hemocue (Ängelhom, Suécia), utilizando-se gota de sangue total obtida imediatamente após punção venosa. Para avaliação das reservas orgânicas de ferro utilizou-se de imunoensaio enzimático (ELISA), pelos reagentes disponíveis comercialmente (Ramco Laboratories, Stafford, Estados Unidos). Esta análise foi realizada em duas etapas: no primeiro momento determinaram-se os níveis plasmáticos de ferritina, e, para as crianças com valores superiores ou iguais a $12 \mu \mathrm{g} / \mathrm{L}$ para este parâmetro, foram posteriormente dosados os níveis plasmáticos dos receptores solúveis de transferrina. Tal estratégia objetivou diagnosticar a deficiência de ferro entre as crianças com processos inflamatórios ou infecciosos, que tendem a elevar os níveis de ferritina sem alterar os níveis de receptor solúvel de transferrina 1 . Foram consideradas anêmicas crianças com concentrações de hemoglobina inferiores a $11 \mathrm{~g} /$ $\mathrm{dL}$ e deficientes em ferro aquelas com valores plasmáticos de ferritina inferiores a $12 \mu \mathrm{g} / \mathrm{L} 1$. Como não há consenso quanto ao valor de corte para diagnóstico de deficiência de ferro com base nos níveis plasmáticos de receptor solúvel de transferrina 1 , empregou-se aquele sugerido pelo fabricante do kit utilizado $(8,3 \mathrm{mg} / \mathrm{L})$. A anemia ferropriva foi definida com base na análise combinada dos três indicadores: concentração de hemoglobina inferior a $11 \mathrm{~g} / \mathrm{dL}$, ferritina plasmática inferior a $12 \mu \mathrm{g} / \mathrm{L}$ e receptor solúvel de transferrina $>8,3 \mathrm{mg} / \mathrm{L}$.

Dupla digitação e análise de consistência dos dados foram realizadas no programa Epi Info 6.04 (Centers for Disease Control and Prevention, Atlanta, Estados Unidos) e as demais análises estatísticas foram conduzidas no programa Stata 9.2 (Stata Corp., College Station, Estados Unidos). Foram calculadas freqüências absolutas, médias e desvios-padrão das variáveis. As proporções foram comparadas pelo teste do quiquadrado, nível de significância de $\mathrm{p}<0,05$. Modelos de regressão múltipla foram analisados para três desfechos separadamente: anemia, anemia ferropriva e deficiência de ferro. Foram investigadas as seguintes variáveis independentes: características demográficas, sócio-econômicas, ambientais, antecedentes maternos de história reprodutiva, características ao nascimento, aleitamento materno, morbidades pregressas, parasitose e antropometria. Na primeira etapa, as associações entre os desfechos e as variáveis independentes foram investigadas com base nas razões de prevalências brutas e respectivos intervalos de $95 \%$ de confiança, selecionando-se associações com valores de $\mathrm{p}<0,20$ (teste de qui- quadrado para heterogeneidade e de tendência linear). Em seguida, as variáveis independentes selecionadas foram estudadas mediante análise múltipla e hierarquizada em regressão de Poisson (variância robusta), utilizando-se modelo conceitual adaptado de Neuman et al. 8 (Figura 1). Sexo, idade e município foram introduzidos nos modelos desde o início. As análises múltiplas foram iniciadas do bloco distal para o proximal. Internamente a cada bloco, foram introduzidas todas as variáveis selecionadas na primeira etapa. As variáveis que apresentaram valores de $\mathrm{p}<0,05$ pelo teste de Wald ou tendência linear foram selecionadas como fator associado aos desfechos e permaneceram nos modelos nas análises dos blocos subseqüentes. As variáveis que apresentaram valores de $\mathrm{p}>0,05$ foram retiradas do modelo e reavaliadas uma a uma quanto a alterações na magnitude das razões de prevalência das variáveis que permaneceram no modelo. Se ocorressem alterações nas magnitudes acima de $10 \%$, a variável era novamente inserida e mantida nos modelos múltiplos finais.

\section{Resultados}

\section{Caracterização da população de estudo}

A população de estudo foi constituída por 624 crianças que completaram os exames físicos e clínicos (92,7\% dos elegíveis). Os pais ou responsáveis por quatro crianças $(0,6 \%)$ não concordaram com a participação no estudo.

A idade média (DP) das crianças estudadas foi de 33,1 meses $(15,5)$, sendo $50,2 \%$ pertencentes ao sexo feminino. Quanto às condições de nascimento, a prevalência de peso ao nascer inferior a $2.500 \mathrm{~g}$ foi de $7,3 \%$. O aleitamento materno foi iniciado para a maioria $(97,5 \%)$, mas a prevalência de aleitamento materno exclusivo até o sexto mês foi de 33,6\%, observando-se mediana de duração do aleitamento materno total de 180 dias. A média de escolaridade formal dos pais (5,9 $\pm 3,8$ anos) ilustrou situação de ensino fundamental incompleto. A maioria das mães era doméstica (67,1\%), e pequena proporção das famílias possuía terra própria (21,8\%). A coleta pública do lixo era realizada na maioria dos domicílios (82\%), no entanto era inexistente rede pública de coleta de esgoto nos municípios, encontrando-se esgoto a céu aberto em $22,3 \%$ dos domicílios.

As prevalências gerais de déficits nutricionais segundo os índices P/E, P/I e E/I foram de $4,2 \%, 6,8 \%$ e 10,2\%, respectivamente. Excesso de peso foi verificado em $3 \%$ das crianças para o índice P/E. Dentre as 513 crianças que tiveram amostra fecal colhida e examinada, 176 (34,3\%) 

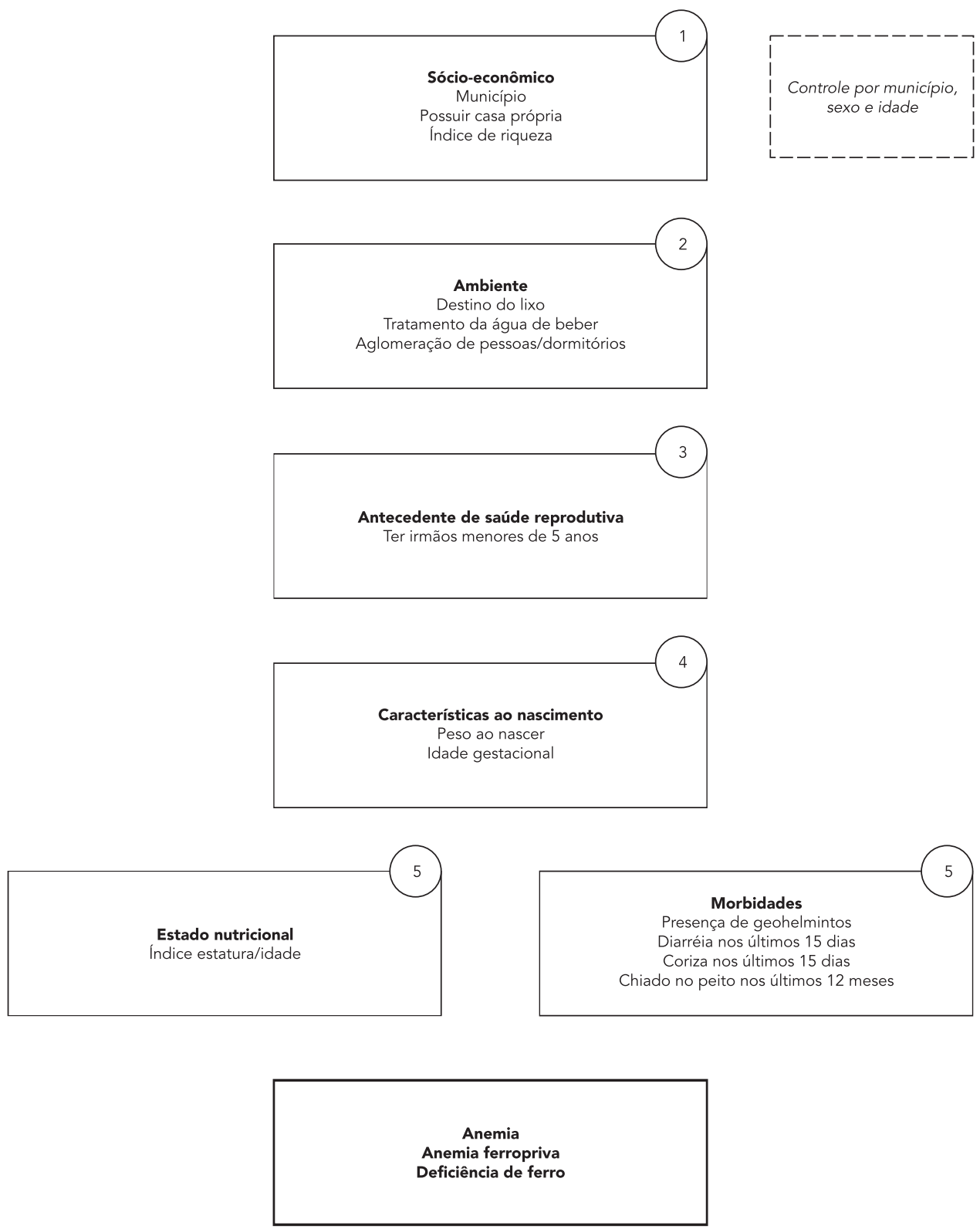

Adaptado de Neuman et al. 8 .

albergavam um ou mais protozoários ou helmintos intestinais. Os parasitas mais comuns foram a Giardia duodenalis $(26,1 \%)$, Entamoeba coli $(5,8 \%)$ e A. lumbricoides (3,3\%). Foi observada baixa prevalência de geohelmintos nesta população (4,9\%) (dados não apresentados em tabela).
Prevalência de anemia, deficiência de ferro e anemia ferropriva

As concentrações de hemoglobina sanguínea foram determinadas para 617 crianças $(98,9 \%$ das entrevistadas e examinadas) e as de ferritina plasmática para 596 (95,5\% das entrevistadas 
e examinadas). Níveis plasmáticos de receptor de transferrina foram determinados para as 304 crianças que apresentaram valores plasmáticos normais ou elevados de ferritina. Baseado nos valores de hemoglobina, 180 crianças $(29,2 \%)$ foram classificadas como anêmicas. Na análise combinada dos níveis plasmáticos de ferritina e de receptor de transferrina, 268 crianças $(44,9 \%)$ foram classificadas como deficientes em ferro. Anemia ferropriva foi detectada em 127 crianças $(21,5 \%)$. Entre as anêmicas, $68,1 \%$ dos casos foram atribuídos à deficiência de ferro. Entre as crianças com deficiência de ferro, 47,6\% apresentavam anemia. À época de realização da pesquisa 24 crianças $(3,8 \%)$ faziam uso de sulfato ferroso.

A Tabela 1 apresenta as prevalências de anemia, anemia ferropriva e deficiência de ferro, de acordo com a faixa etária e o sexo. Houve diferença estatisticamente significante entre as prevalências dos três desfechos segundo faixa etária, sendo elas maiores entre os menores de 24 meses. Não se verificaram diferenças de prevalências entre os sexos.

Fatores associados à anemia, anemia ferropriva e deficiência de ferro

As prevalências de anemia, anemia ferropriva e deficiência de ferro entre as categorias das variáveis independentes, assim como as razões de prevalências brutas (intervalo de $95 \%$ de confiança) entre os desfechos e variáveis independentes são apresentadas nas Tabelas 2 e 3. Maiores riscos para os três desfechos relacionaram-se às seguintes variáveis: pertencer ao Município de Assis Brasil, ser do sexo masculino, idade inferior a 48 meses, não possuir casa própria, pertencer ao menor tercil do índice de riqueza, aglomeração de pessoas/dormitório superior a 4,5, prematuridade, pertencer ao menor quartil do índice estatura/idade e infestação por geohelmintos. O não-tratamento da água de beber, o peso ao nascer inferior a $2.500 \mathrm{~g}$ e ocorrências de diarréia e coriza nos últimos 15 dias e de chiado no peito nos últimos 12 meses foram associados à anemia e anemia ferropriva. Criança ter irmãos menores de cinco anos mostrou-se associado à anemia e o lançamento do lixo em áreas abertas associou-se à anemia e à deficiência de ferro.

A Tabela 4 apresenta os fatores associados aos desfechos nos modelos múltiplos finais. Crianças com idade inferior a 24 meses estavam pelo menos duas vezes em maior risco para anemia, anemia ferropriva e deficiência de ferro, observando-se relação linear de aumento das prevalências dos distúrbios com a redução da idade. Sexo foi determinante apenas da deficiência de ferro nesta população, conferindo risco $19 \%$ maior entre os meninos. O índice de riqueza mostrou-se associado, também de forma linear, à anemia ferropriva; crianças localizadas no terceiro tercil da distribuição mostraram-se 38\% mais protegidas em relação àquelas do primeiro tercil. De forma linear para os desfechos de anemia e anemia ferropriva, as crianças localizadas no segundo quartil ou maior de distribuição do índice E/I estavam pelo menos $36 \%$ mais protegidas dos distúrbios, enquanto a recente ocorrência de diarréia ofereceu $47 \%$ maior risco para anemia e $44 \%$ para anemia ferropriva.

A infestação por geohelmintos conferiu risco para os três desfechos. Crianças com o parasita estavam em $45 \%$ maior risco para a deficiência

Prevalência de anemia, anemia ferropriva e deficiência de ferro entre as crianças de 6 a 60 meses de acordo com sexo e faixa etária. Municípios de Assis Brasil e Acrelândia, Acre, Brasil.

\begin{tabular}{|c|c|c|c|c|c|c|}
\hline \multirow[t]{2}{*}{ Idade (meses) } & \multicolumn{2}{|c|}{$\begin{array}{l}\text { Anemia * } \\
\text { n (\%) }\end{array}$} & \multicolumn{2}{|c|}{$\begin{array}{c}\text { Anemia ferropriva * } \\
\text { n (\%) }\end{array}$} & \multicolumn{2}{|c|}{$\begin{array}{c}\text { Deficiência de Ferro * } \\
\text { n (\%) }\end{array}$} \\
\hline & Meninos & Meninas & Meninos & Meninas & Meninos & Meninas \\
\hline $6-11,9$ & $17(44,7)$ & $18(52,9)$ & $8(25,8)$ & $10(31,3)$ & $12(38,7)$ & $15(46,9)$ \\
\hline $12-23,9$ & $33(58,9)$ & $31(44,9)$ & $29(52,7)$ & $25(37,9)$ & $45(78,9)$ & $43(65,2)$ \\
\hline $24-35,9$ & $25(35,7)$ & $19(26,0)$ & $21(30,4)$ & $15(22,1)$ & $41(59,4)$ & $31(44,9)$ \\
\hline $36-60$ & $21(14,9)$ & $14(10,5)$ & $11(8,2)$ & $7(5,3)$ & $45(32,4)$ & $33(25,2)$ \\
\hline Valor de $p^{\star \star}$ & 0,000 & 0,000 & 0,000 & 0,000 & 0,000 & 0,000 \\
\hline
\end{tabular}

* $n=617$ crianças com dosagem de hemoglobina, $n=596$ crianças com dosagem de ferritina e $n=589$ crianças com

dosagem de hemoglobina e ferritina plasmática;

** Teste do qui-quadrado: comparação de proporções segundo faixa etária. 
Prevalências de anemia, anemia ferropriva e deficiência de ferro entre as categorias das variáveis independentes e razões de prevalências brutas (RP) e respectivos intervalos de 95\% de confiança (IC95\%) entre os desfechos e as variáveis demográficas, sócio-econômicas, ambientais e antecedentes de saúde reprodutiva. Municípios de Assis Brasil e Acrelândia, Acre, Brasil.

\begin{tabular}{|c|c|c|c|c|c|c|c|c|c|}
\hline Variáveis * & \multicolumn{3}{|c|}{ Anemia } & \multicolumn{3}{|c|}{ Anemia ferropriva } & \multicolumn{3}{|c|}{ Deficiência de ferro } \\
\hline$\geq 48$ & 131 & 13,0 & 1,00 & 128 & 4,7 & 1,00 & 130 & 26,1 & 1,00 \\
\hline $24-48$ & 286 & 21,7 & 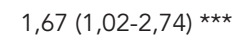 & 275 & 17,5 & $3,72(1,63-8,48) * \star \star$ & 278 & 41,7 & $1,59(1,16-2,20) * \star \star$ \\
\hline $6-24$ & 197 & 50,2 & 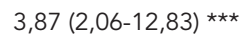 & 184 & 39,1 & $8,38(3,74-18,62)$ *** & 186 & 61,8 & $2,36(1,73-3,22)$ *** \\
\hline \multicolumn{10}{|l|}{ Sexo } \\
\hline Masculino & 305 & 31,5 & 1,00 & 290 & 23,8 & 1,00 & 296 & 48,3 & 1,00 \\
\hline Feminino & 312 & 26,9 & $0,85(0,67-1,09)$ & 299 & 19,4 & $0,81(0,60-1,11) * *$ & 300 & 41,3 & $0,85(0,71-1,02)$ ** \\
\hline Sem informação & 0 & - & - & 0 & - & - & 0 & - & - \\
\hline \multicolumn{10}{|l|}{ Município } \\
\hline $\operatorname{Sim}$ & 487 & 27,8 & 1,00 & 469 & 19,9 & 1,00 & 476 & 43,7 & 1,00 \\
\hline Não & 125 & 32,7 & 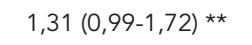 & 116 & 27,9 & $1,60(1,15-2,22) * \star \star$ & 116 & 50,4 & 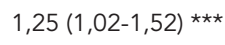 \\
\hline Sem informação & 5 & - & - & 4 & - & - & 4 & - & - \\
\hline \multicolumn{10}{|l|}{ Índice de riqueza em escore z } \\
\hline Tercil 1 & 195 & 33,3 & 1,00 & 183 & 27,9 & 1,00 & 183 & 48,6 & 1,00 \\
\hline Tercil 2 & 204 & 33,3 & $1,00(0,76-1,32)$ & 199 & 21,6 & $0,77(0,54-1,10) * \star$ & 204 & 45,6 & $0,94(0,76-1,16)$ \\
\hline Tercil 3 & 212 & 21,2 & $0,64(0,46-0,88) * \star \star$ & 202 & 15,8 & $0,57(0,38-0,84) * \star \star$ & 204 & 40,7 & $0,84(0,67-1,04) * \star$ \\
\hline Sem informação & 6 & - & - & 5 & - & - & 5 & - & - \\
\hline \multicolumn{10}{|l|}{ Aglomeração de pessoas } \\
\hline \multicolumn{10}{|l|}{ por dormitório } \\
\hline Joga em área aberta/rio & 46 & 42,3 & 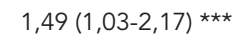 & 43 & 23,2 & $1,08(0,61-1,191)$ & 43 & 55,8 & $1,26(0,95-1,68)$ ** \\
\hline Sem informação & 8 & - & - & 6 & - & - & 6 & - & - \\
\hline \multicolumn{10}{|l|}{ Tratamento da água de beber } \\
\hline $\operatorname{Sim}$ & 429 & 26,3 & 1,00 & 415 & 19,3 & 1,00 & 421 & 43,5 & 1,00 \\
\hline Não & 183 & 36,1 & $1,37(1,07-1,76) * \star \star$ & 170 & 27,6 & $1,43(1,05-1,96)$ *** & 171 & 48,5 & $1,11(0,92-1,35)$ \\
\hline Sem informação & 5 & - & - & 4 & - & - & 4 & - & - \\
\hline \multicolumn{10}{|l|}{ A criança tem irmãos menores } \\
\hline \multicolumn{10}{|l|}{ de 5 anos } \\
\hline Não & 282 & 25,5 & 1,00 & 267 & 19,5 & 1,00 & 269 & 45,7 & 1,00 \\
\hline $\operatorname{Sim}$ & 329 & 32,5 & $1,27(0,99-1,64) \star \star$ & 317 & 23,6 & $1,21(0,89-1,66)$ & 322 & 44,4 & $0,97(0,81-1,16)$ \\
\hline Sem informação & 6 & - & - & 5 & - & - & 5 & - & - \\
\hline
\end{tabular}

* Número total de observações diferem ( $n=617$ para anemia, $n=589$ para anemia ferropriva e $n=596$ para deficiência de ferro) devido à ausência de informações:

** $p>0,05$ e $<0,20$;

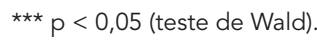


Prevalências de anemia, anemia ferropriva e deficiência de ferro entre as categorias das variáveis independentes e razões de prevalências brutas (RP) e respectivos intervalos de $95 \%$ de confiança (IC95\%) entre os desfechos e as variáveis de antecedentes maternos de saúde reprodutiva, características ao nascimento, morbidades e antropometria. Acre, Brasil.

\begin{tabular}{|c|c|c|c|c|c|c|c|c|c|}
\hline \multirow[t]{2}{*}{ Variáveis * } & \multicolumn{3}{|r|}{ Anemia } & \multicolumn{3}{|c|}{ Anemia ferropriva } & \multicolumn{3}{|c|}{ Deficiência de ferro } \\
\hline & $\mathrm{n}$ & $\%$ & RP (IC95\%) & $n$ & $\%$ & RP (IC95\%) & 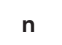 & $\%$ & RP (IC95\%) \\
\hline \multicolumn{10}{|l|}{ Idade gestacional } \\
\hline A termo & 569 & 28,3 & 1,00 & 542 & 20,8 & 1,00 & 548 & 44,3 & 1,00 \\
\hline Prematuro & 15 & 46,7 & $1,65(0,94-2,88) * \star$ & 15 & 46,7 & 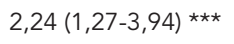 & 15 & 60,0 & $1,35(0,88-2,07)$ ** \\
\hline Sem informação & 33 & - & - & 32 & - & - & 33 & - & - \\
\hline \multicolumn{10}{|l|}{ Peso ao nascer $(\mathrm{kg})$} \\
\hline$<2,5$ & 42 & 47,6 & 1,00 & 42 & 33,3 & 1,00 & 42 & 47,6 & 1,00 \\
\hline $2,5-3,0$ & 84 & 32,1 & $0,67(0,43-1,05) * \star$ & 81 & 24,7 & $0,74(0,42-1,31)$ & 82 & 42,7 & $0,90(0,60-1,34)$ \\
\hline$>3,0$ & 444 & 26,6 & $0,56(0,39-0,79) * \star \star *$ & 421 & 19,9 & $0,60(0,37-0,96) * * *$ & 427 & 45,4 & $0,95(0,68-1,33)$ \\
\hline Sem informação & 47 & - & - & 45 & - & - & 45 & - & - \\
\hline \multicolumn{10}{|l|}{ Estatura-idade em escore z } \\
\hline Quartil 1 & 163 & 36,2 & 1,00 & 155 & 27,1 & 1,00 & 155 & 46,4 & 1,00 \\
\hline Quartil 2 & 154 & 30,5 & $0,84(0,62-1,15)$ & 150 & 22,0 & $0,81(0,54-1,21)$ & 155 & 45,8 & $0,99(0,77-1,25)$ \\
\hline Quartil 3 & 147 & 25,2 & $0,69(0,49-0,98) * \star \star$ & 140 & 17,1 & $0,63(0,40-0,99) * \star \star$ & 142 & 38,7 & $0,83(0,64-1,09) * *$ \\
\hline Quartil 4 & 145 & 23,4 & $0,65(0,45-0,93) * \star \star$ & 137 & 19,7 & $0,73(0,47-1,11) * \star$ & 137 & 48,2 & $1,04(0,81-1,32)$ \\
\hline Sem informação & 8 & - & - & 7 & - & - & 7 & - & - \\
\hline \multicolumn{10}{|l|}{ Presença de geohelmintos \# } \\
\hline Não & 483 & 27,3 & 1,00 & 466 & 19,7 & 1,00 & 471 & 42,9 & 1,00 \\
\hline Sim & 23 & 43,5 & $1,59(0,97-2,59)$ ** & 20 & 35,0 & $1,77(0,95-3,31) * \star$ & 22 & 63,6 & $1,48(1,06-2,07) * \star \star$ \\
\hline Sem informação & 111 & - & - & 103 & - & - & 103 & - & - \\
\hline \multicolumn{10}{|l|}{ Diarréia nos últimos 15 dias } \\
\hline Não & 433 & 24,7 & 1,00 & 413 & 18,2 & 1,00 & 416 & 43,7 & 1,00 \\
\hline Sim & 181 & 40,3 & 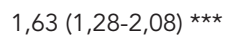 & 173 & 30,0 & 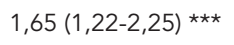 & 177 & 46,9 & $1,07(0,88-1,30)$ \\
\hline Sem informação & 3 & - & - & 3 & - & - & 3 & - & - \\
\hline \multicolumn{10}{|l|}{ Coriza nos últimos 15 dias } \\
\hline Não & 316 & 25,9 & 1,00 & 303 & 17,8 & 1,00 & 307 & 45,3 & 1,00 \\
\hline Sim & 295 & 33,2 & $1,28(1,00-1,64) * *$ & 280 & 26,1 & $1,46(1,07-1,99) * \star \star$ & 283 & 44,5 & $0,98(0,82-1,18)$ \\
\hline Sem informação & 6 & - & - & 6 & - & - & 6 & - & - \\
\hline \multicolumn{10}{|c|}{ Pneumonia nos últimos 12 meses } \\
\hline Não & 513 & 28,5 & 1,00 & 489 & 20,8 & 1,00 & 496 & 44,1 & 1,00 \\
\hline $\operatorname{Sim}$ & 91 & 33,0 & $1,16(0,84-1,60)$ & 87 & 26,4 & $1,27(0,86-1,87)$ & 87 & 48,3 & $1,09(0,86-1,39)$ \\
\hline Sem informação & 13 & - & - & 13 & - & - & 13 & - & - \\
\hline \multicolumn{10}{|c|}{ Chiado no peito nos últimos 12 meses } \\
\hline Não & 492 & 26,0 & 1,00 & 471 & 18,7 & 1,00 & 476 & 43,9 & 1,00 \\
\hline $\operatorname{Sim}$ & 121 & 42,9 & 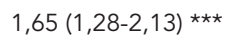 & 114 & 34,2 & $1,83(1,33-2,51) * \star \star$ & 116 & 48,3 & $1,10(0,89-1,36)$ \\
\hline Sem informação & 4 & - & - & 4 & - & - & 4 & - & - \\
\hline \multicolumn{10}{|c|}{ Duração aleitamento materno (dias) } \\
\hline$>120$ & 224 & 29,0 & 1,00 & 217 & 20,3 & 1,00 & 218 & 45,9 & 1,00 \\
\hline $30-120$ & 250 & 31,2 & $1,06(0,81-1,40)$ & 235 & 23,0 & $1,13(0,80-1,61)$ & 238 & 44,9 & $0,98(0,80-1,20)$ \\
\hline$<30$ ou nunca mamou & 93 & 23,6 & $0,81(0,54-1,24)$ & 88 & 20,4 & $1,00(0,80-1,61)$ & 91 & 45,0 & $0,98(0,75-1,28)$ \\
\hline Sem informação & 50 & - & - & 49 & - & - & 42 & - & - \\
\hline
\end{tabular}

* Número total de observações diferem ( $n=617$ para anemia, $n=589$ para anemia ferropriva e $n=596$ para deficiência de ferro) devido à ausência de informações;

** $\mathrm{p}>0,05$ e $<0,20$;

$\star \star \star ~ p<0,05$ (teste de Wald);

\# Os geohelmintos desta população incluíram: Ancylostoma duodenale (0,4\%), Ascaris lumbricoides (3,3\%), Strongyloides stercoralis (0,2\%) e Trichuris trichura $(1,8 \%)$. 
Fatores associados à anemia, anemia ferropriva e deficiência de ferro entre crianças de 6 a 60 meses. Municípios de Assis Brasil e Acrelândia, Acre, Brasil.

\begin{tabular}{|c|c|c|c|}
\hline Variáveis & $\begin{array}{c}\text { Anemia * } \\
\text { RP ajustada (IC95\%) }\end{array}$ & $\begin{array}{l}\text { Anemia ferropriva ** } \\
\text { RP ajustada (IC95\%) }\end{array}$ & $\begin{array}{l}\text { Deficiência de ferro } \star \star \star \\
\text { RP ajustada (IC95\%) }\end{array}$ \\
\hline \multicolumn{4}{|l|}{ Idade (meses) } \\
\hline$\geq 48$ & 1,00 & 1,00 & 1,00 \\
\hline $24-48$ & $1,44(0,86-2,41)$ & $2,73(1,20-6,22)$ & $1,41(1,00-1,99)$ \\
\hline $6-24$ & $3,76(2,32-6,10)$ & $6,87(3,10-15,22)$ & $2,14(1,54-2,98)$ \\
\hline Valor de $p$ de tendência linear & 0,000 & 0,000 & 0,000 \\
\hline \multicolumn{4}{|l|}{ Sexo } \\
\hline Masculino & - & - & 1,00 \\
\hline Feminino & - & - & $0,80(0,66-0,98)$ \\
\hline Valor de $p$ de tendência linear & - & - & - \\
\hline \multicolumn{4}{|l|}{ Índice de riqueza em escore z } \\
\hline Tercil 1 & 1,00 & 1,00 & - \\
\hline Tercil 2 & $1,12(0,83-1,51)$ & $0,91(0,63-1,32)$ & - \\
\hline Tercil 3 & $0,77(0,53-1,10)$ & $0,62(0,40-0,98)$ & - \\
\hline Valor de $p$ de tendência linear & 0,278 & 0,041 & - \\
\hline \multicolumn{4}{|l|}{ Aglomeração pessoas no dormitório } \\
\hline$\leq 4,5$ & 1,00 & 1,00 & - \\
\hline$>4,5$ & $1,30(0,90-1,87)$ & $1,27(0,77-2,09)$ & - \\
\hline \multicolumn{4}{|l|}{ Índice Estatura/ldade em escore z } \\
\hline Quartil 1 & 1,00 & 1,00 & - \\
\hline Quartil 2 & $0,62(0,44-0,86)$ & $0,51(0,33-0,79)$ & - \\
\hline Quartil 3 & $0,64(0,44-0,92)$ & $0,54(0,34-0,86)$ & - \\
\hline Quartil 4 & $0,49(0,34-0,71)$ & $0,56(0,36-0,86)$ & - \\
\hline Valor de $p$ de tendência linear & 0,000 & 0,014 & - \\
\hline \multicolumn{4}{|l|}{ Presença de geohelmintos } \\
\hline Não & 1,00 & 1,00 & 1,00 \\
\hline Sim & $1,61(0,92-2,80)$ & $1,54(0,81-2,92)$ & $1,43(1,02-2,00)$ \\
\hline \multicolumn{4}{|l|}{ Diarréia nos últimos 15 dias } \\
\hline Não & 1,00 & 1,00 & - \\
\hline Sim & $1,47(1,12-1,92)$ & $1,44(1,03-2,01)$ & - \\
\hline
\end{tabular}

* Modelo $n=490$. Ajustado por sexo e município;

** Modelo $n=472$. Ajustado por sexo e município;

*** Modelo $n=492$. Ajustado por município.

de ferro. Para os modelos de anemia e anemia ferropriva, a exclusão desta variável interferiu em mais de $10 \%$ nas razões de prevalências das variáveis, permanecendo nos modelos finais como associadas aos dois desfechos.

\section{Discussão}

A prevalência de anemia entre as crianças de 6 a 60 meses nos municípios acreanos estudados caracteriza esta carência como um problema de saúde pública moderado na região 1 . Toda- via, entre os anêmicos, 31,9\% não apresentaram evidências bioquímicas de deficiência de ferro, sugerindo outros determinantes da anemia para tais casos. Crianças com idade inferior a 24 meses e com infestação por geohelmintos estavam em maior risco para anemia, anemia ferropriva e deficiência de ferro. Menores valores do índice estatura/idade e acometimento recente de diarréia conferiram maior risco para anemia e anemia ferropriva. Pertencer ao terceiro quartil do índice de riqueza conferiu proteção à anemia ferropriva, e ser do sexo feminino foi protetor frente à deficiência de ferro. 
O Estado do Acre apresenta municípios com pequenas populações, de forte característica rural. O índice de desenvolvimento infantil (IDI) do estado era o segundo pior do país em 2001 17, sendo as taxas de mortalidade infantil estimadas, naquele ano, de 67,4/1.000 e de 70,75/1.000 nascidos vivos em Assis Brasil e Acrelândia, respectivamente, superiores à média nacional $(27,7 / 1.000$ nascidos vivos) 18 . No presente estudo, constatou-se baixo nível de escolaridade formal dos pais, altas taxas de desemprego materno e pequeno número de famílias possuidoras de terras próprias. Foi registrada ausência de rede coletora de esgoto nos municípios e a presença dele fluindo a céu aberto para quase 1/5 dos domicílios. Quanto à situação de saúde na infância, esta população se caracterizou por prevalência de baixo peso ao nascer ligeiramente superior à média nacional $(6,6 \%) 3$, com prevalência de crianças que iniciaram o aleitamento materno próxima à media nacional 3 . Apesar da maior prevalência de aleitamento materno exclusivo e total entre as crianças acreanas, comparadas àquelas da pesquisa nacional 3 , os valores ainda estão distantes do preconizado pela OMS 19.

No presente estudo, destaca-se que cerca de $1 / 3$ dos casos de anemia não foram devidos à deficiência de ferro, corroborando a premissa da OMS de não se utilizar isoladamente o indicador de concentração de hemoglobina sanguínea como teste de rastreamento para deficiência de ferro 1. Limitações de nosso estudo relacionamse à não-avaliação do consumo alimentar e dos valores séricos de outros micronutrientes como ácido fólico, vitamina $\mathrm{B}_{12}$ e retinol, que poderia complementar informações acerca da gênese da anemia não explicada pela deficiência de ferro.

As respectivas prevalências, nacional e na Região Norte, para anemia entre menores de cinco anos, de acordo com a Pesquisa Nacional de Demografia e Saúde (PNDS-2006) ${ }^{3}$, foram de $20,9 \%$ e de $10,4 \%$. Contudo, a prevalência de anemia observada no presente estudo foi inferior às estimativas encontradas em outros estudos populacionais brasileiros (40,9\% no Estado de Pernambuco 20; 46,9\% no Município de São Paulo 4; $36,4 \%$ no Estado da Paraíba 5; 46,4\% em Salvador 6 e $45 \%$ no Estado de Alagoas 7). Prevalências também superiores de anemia foram detectadas em crianças menores de três anos do sul do Brasil (54\% em Criciúma 8 e 47,8\% em Porto Alegre 9). Porém, alguns fatores restringem a validade de comparações da prevalência com os estudos citados, uma vez que estes foram derivados de levantamentos com amostras sanguíneas colhidas por punção digital, procedimento que pode levar à diluição da amostra e introduzir erro sistemático nas dosagens de hemoglobina ${ }^{21}$, além de possíveis variações de tendência temporal. Em relação às prevalências de anemia ferropriva e deficiência de ferro, há escassez de estudos brasileiros de base populacional que permitam a comparação das prevalências encontradas em nosso estudo, sendo localizado um único estudo anterior conduzido na Amazônia Ocidental brasileira. Tratou-se de estudo prospectivo de incidência de anemia e malária entre os habitantes de todas as faixas etárias da área rural de Urupá, distrito de Porto Velho, Rondônia. O levantamento inicial, que envolveu 133 indivíduos, dos quais $17,7 \%$ eram menores de seis anos, identificou prevalências gerais de anemia, deficiência de ferro e anemia ferropriva de $10 \%, 10 \%$ e $5,4 \%$, respectivamente 22 . Na América Latina, estudos realizados na Argentina apontam que a deficiência de ferro é um problema de saúde pública entre os menores de 24 meses 23,24. Estudo mais recente, no Chaco, neste mesmo país, apontou que a deficiência de ferro (concentração de ferritina plasmática $<12 \mu \mathrm{g} / \mathrm{L}$ ) variou de $36,6 \%$ entre crianças de 6 -8 meses a $72,9 \%$ entre aquelas de 18 a 24 meses 24 .

O estudo dos determinantes da anemia, anemia ferropriva e deficiência de ferro apontou que crianças na faixa de 6 a 24 meses estavam em maior risco para os três desfechos, com redução das prevalências com o aumento da idade. Este comportamento foi apontado em outros estudos que investigaram anemia 4,8,20 e deficiência de ferro 25 . Isso se explica pelo efeito independente da idade sobre os indicadores hematológicos de ferro de crianças mais jovens, que têm maior necessidade fisiológica do mineral em razão da maior taxa de crescimento 25 . Não obstante, nessa faixa etária, a baixa duração do aleitamento materno exclusivo e a introdução precoce de alimentos de baixa densidade de ferro biodisponível ${ }^{1}$ somam efeito multiplicativo para aumento do risco de anemia e deficiência de ferro. Em nosso estudo, crianças do sexo feminino estavam mais protegidas contra a deficiência de ferro, corroborando outros estudos que apontaram maior risco de anemia e deficiência de ferro entre crianças do sexo masculino 26,27, que se explica, em parte, por diferenças genéticas, de velocidade de crescimento e de práticas alimentares 28.

No nível distal de determinação, pertencer ao maior tercil do índice de riqueza foi protetor para a anemia ferropriva, não se mostrando associado com as demais carências (anemia e deficiência de ferro). Esta última observação concorda com resultados recentes para crianças de 6 a 59 meses da PNDS-2006 ${ }^{3}$, em que não se encontraram prevalências estatisticamente diferentes de anemia nos diferentes estratos econômicos (A e B, C, D e E), mas difere do verificado em estudos 
populacionais pontuais conduzidos no início dos anos 2000 4,8,9, com maiores prevalências de anemia entre crianças mais pobres.

No presente estudo, houve associação entre menores valores para o índice estatura/idade com anemia e anemia ferropriva. Osório et al. 20, avaliando menores de cinco anos, no Estado de Pernambuco, e Lima et al. 29 , estudando crianças de 12 meses na Zona da Mata Meridional deste mesmo estado, apontaram também associação positiva entre valores do índice E/I e de hemoglobina. Não é possível estabelecer, com base no presente estudo, de natureza transversal, fatores de causalidade entre menores valores para o índice E/I e a ocorrência de anemia e anemia ferropriva. De fato, a anemia afeta o crescimento e desenvolvimento físico de crianças, podendo levar ao déficit estatural 30, assim como, a desnutrição pode alterar a concentração de hemoglobina como mecanismo adaptativo por conta da privação dietética 31 . No presente estudo, o acometimento recente de diarréia foi determinante da anemia e anemia ferropriva. Observação semelhante foi apontada para crianças de 6 a 59 meses do Timor Leste 32 , onde menores valores de hemoglobina sanguínea foram verificados em crianças com relato de diarréia nas últimas duas semanas prévias ao estudo. $\mathrm{O}$ aumento no risco de anemia com aumento de infecções diarréicas e respiratórias também foi verificado por Levy et al. 33 , em estudo prospectivo com beduínos de 18 meses. De acordo com Beresford et al. ${ }^{34}$, a diarréia pode ocasionar perda sanguínea intestinal e a ocorrência recente dessa infecção tem sido sugerida como um marcador de episódios mais freqüentes e persistentes. Por causa do delineamento transversal do presente estudo, a relação temporal entre infecções e risco para anemia e anemia ferropriva não pôde ser verificada. Além disso, na literatura, a relação causal entre estado nutricional de ferro e suscetibilidade a infecções permanece controversa pela escassez de evidências provenientes de estudos longitudinais. Outro fato adicional é que esta relação pode ser confundida também por variáveis ambientais e sócio-econômicas, visto que elas são fatores de risco tanto para a anemia e anemia ferropriva, quanto para infecções 35 .

Apesar da baixa prevalência de infecção por geohelmintos neste estudo, foi verificada sua associação com os três desfechos estudados, contrariamente ao observado por Tatala et al. 2 em estudo realizado com pré-escolares da Tanzânia 24 , onde foi observada prevalência de geohelmintos semelhante à verificada entre as crianças de nosso estudo (3,8\%), entretanto sem associação com a anemia. Estudo conduzido com maiores de cinco anos, adolescentes e adultos em área rural do Acre, próximo à Acrelândia, apontou prevalência de geohelmintos de $11,6 \%$, observando correlação negativa dessa infecção com os valores de ferritina sérica 12 . É classicamente apontada a ocorrência de anemia pela espoliação sanguínea intestinal por geohelmintos 36 . Pela exposição à situação ambiental desfavorável entre as crianças deste estudo, era de se esperar maior prevalência de infestação parasitária. Uma possível explicação para esta constatação talvez possa ser sustentada pelo uso rotineiro de medicamentos anti-helmínticos administrados pelos agentes de saúde do PSF dos municípios à época da pesquisa. Mesmo assim, infecções por protozoários intestinais foram mais prevalentes entre as crianças do Acre do que entre crianças menores de cinco anos do Município de São Paulo ${ }^{37}$, o que pode ter contribuído para a elevada ocorrência de doença diarréica entre as crianças deste estudo, com valores substancialmente maiores ao registrado para menores de cinco anos de São Paulo $(4,7 \%) 38$.

Os resultados aqui apresentados apontaram que a anemia, anemia ferropriva e deficiência de ferro afetaram especialmente os menores de 24 meses, associando-se com o menor crescimento estatural e infecções diarréicas e por geohelmintos. Dessa forma, intervenções voltadas prioritariamente aos menores de dois anos, que busquem garantir adequado crescimento e controle de infecções, tornam-se necessárias, o que, na prática, pode ser revertido em melhorias dos serviços de pré-natal e puericultura nos municípios. 


\section{Resumo}

Estudo transversal de base populacional que investigou prevalênciasde anemia e fatores associados à anemia, anemia ferropriva e deficiência de ferro entre crianças de 6 a 60 meses da área urbana de dois municípios do Acre, Brasil $(N=624)$. Dosagens de hemoglobina sanguínea, ferritina e receptor solúvel de transferrina plasmáticas foram realizadas mediante sangue venoso. Condições sócio-econômicas, demográficas e de morbidade foram obtidas por questionário. Razões de prevalências foram calculadas por regressão de Poisson em modelo hierárquico. As prevalências de anemia, anemia ferropriva e deficiência de ferro foram de 30,6\%, 20,9\% e 43,5\%, respectivamente. Menores de 24 meses apresentaram maior risco para anemia, anemia ferropriva e deficiência de ferro. Pertencer ao maior tercil do indice de riqueza conferiu proteção contra anemia ferropriva $(R P=0,62 ;$ IC95\%: 0,40-0,98). Pertencer ao maior quartil do indice estatura/idade foi protetor contra anemia $(0,62 ; 0,44-0,86)$ e anemia ferropriva $(0,51 ; 0,33-0,79)$, e ocorrência recente de diarréia representou risco (anemia: 1,47; 1,121,92 e anemia ferropriva: 1,44; 1,03-2,01). A infestação por geohelmintos conferiu risco para anemia, anemia ferropriva e deficiência de ferro.

Anemia; Deficiência de Ferro; Bem-Estar da Criança

\section{Colaboradores}

T. G. Castro e M. A. Cardoso participaram do planejamento do estudo, coleta dos dados, análise, interpretação dos resultados, redação inicial e revisão da versão final do manuscrito. M. Silva-Nunes participou da coleta dos dados, exame médico, coleta de sangue e determinação de hemoglobina sanguínea dos participantes do estudo e revisão da redação do manuscrito final. W. L. Conde participou da análise dos dados, interpretação dos resultados e revisão da redação do manuscrito final. P. T. Muniz participou do desenho do estudo, coleta dos dados e revisão da redação do manuscrito final.

\section{Agradecimentos}

Ao Professor Álvaro Vigo, da Universidade Federal do Rio Grande do Sul, por sua orientação nos comandos para análise de regressão de Poisson. Agradecimentos à Francisca Souza Santiago, pelo suporte técnico em campo. Financiamento: Conselho Nacional de Desenvolvimento Científico e Tecnológico (CNPq; processo no ${ }^{\circ}$ 50.2937/2003-3) e bolsa de doutorado da Fundação de Amparo à Pesquisa do Estado de São Paulo (FAPESP; processo $n^{\circ}$. 03/12491-7).

\section{Referências}

1. World Health Organization. Iron deficiency anaemia: assessment, prevention and control. Geneva: World Health Organization; 2001.

2. World Health Organization. Worldwide prevalence of anemia 1993-2005: WHO global database on anemia. Geneva: World Health Organization; 2008.

3. Ministério da Saúde. Pesquisa Nacional de Demografia e Saúde da Criança e da Mulher - relatório final. Brasília: Ministério da Saúde; 2008.

4. Monteiro CA, Szarfac SC, Mondini L. Tendência secular da anemia na infância na cidade de São Paulo (1984-1996). Rev Saúde Pública 2000; 34:62-72.

5. Oliveira RS, Diniz AS, Benigna MJC, Silva SMM, Lola MM, Gonçalves MC, et al. magnitude, distribuição espacial e tendência da anemia em pré-escolares da Paraíba. Rev Saúde Pública 2002; 36:1-11.
6. Assis AMO, Barreto ML, Gomes GSS, Prado MS, Santos NS, Santos LMP, et al. Childhood anemia prevalence and associated factors in Salvador, Bahia, Brasil. Cad Saúde Pública 2004; 20:1633-41.

7. Vieira RCS, Ferreira HS, Costa ACS, Moura FA, Florêncio TMM, Torres ZMC. Prevalência e fatores de risco para anemia em crianças pré-escolares do Estado de Alagoas, Brasil. Rev Bras Saúde Matern Infant 2010; 10:107-16.

8. Neuman NA, Tanaka OY, Szarfac SC, Guimarães PRV, Victora CG. Prevalência e fatores de risco para anemia no sul do Brasil. Rev Saúde Pública 2000; 34:56-63. 
9. Silva LSM, Giugliani ERJ, Aerts DRGC. Prevalência e determinantes de anemia em crianças de Porto Alegre, RS, Brasil. Rev Saúde Pública 2001; 35:66-73.

10. Batista-Filho M, Rissin A. A transição nutricional no Brasil: tendências regionais e temporais. Cad Saúde Pública 2003; 19 Suppl 1:S181-91.

11. Paiva AA, Rondó PHC, Guerra-Shinohara EM. Parâmetros para avaliação do estado nutricional de ferro. Rev Saúde Pública 2000; 34:421-6.

12. Ferreira MU, Silva-Nunes M, Bertolino CN, Malafronte RS, Muniz PT, Cardoso MA. Anemia and iron deficiency in school children, adolescents and adults: a community-based study in Rural Amazonia. Am J Public Health 2007; 97:237-9.

13. Muniz PT, Castro TG, Araújo TS, Nunes NB, SilvaNunes M, Hoffmann EHE, et al. Child health and nutrition in the Western Brazilian Amazon: population-based surveys in two counties in Acre State. Cad Saúde Pública 2007; 23:1283-93.

14. World Health Organization. Physical status: the use and interpretation of anthropometry. Geneva: World Health Organization; 1995.

15. World Health Organization. WHO child growth standards: length/height-for-age, weight-for-age, weight-for-length, weight-for height and body mass index-for-age: methods and development. Geneva: World Health Organization; 2006.

16. Hoffman W, Pons JA, Janer JL. The sedimentation concentration method in schistosomiasis mansoni. PR J Public Health Trop Med 1934; 9:283-91.

17. Fundo das Nações Unidas para a Infância. State of the world's children 2001. http://www.unicef. org/brazil/sib2001/index.html (acessado em 15/ Abr/2005).

18. Ministério da Saúde. Saúde Brasil 2004: uma análise da situação de saúde. Brasília: Ministério da Saúde; 2004

19. World Health Organization. Indicators for assessing breast feeding practices. Geneva: World Health Organization; 1991.

20. Osório MM, Lira PIC, Ashworth A. Factors associated with $\mathrm{Hb}$ concentration in children aged 6-59 months in the State of Pernambuco, Brazil. Br J Nutr 2004; 91:307-14.

21. Sari M, de Pee S, Martini E, Herman S, Sugiatmi, Bloem MW, et al. Estimating the prevalence of anaemia: a comparison of three methods. Bull World Health Organ 2001; 79:506-11.

22. Cardoso MA, Ferreira MU, Camargo LM, Szarfarc SC. Anaemia, iron deficiency and malaria in a rural community in Brazilian Amazon. Eur J Clin Nutr $1994 ; 48: 326-32$

23. Calvo EB, Gnazzo N. Prevalence of iron deficiency in children aged 9-24 mo from a large urban area of Argentina. Am J Clin Nutr 1990; 52:534-40.
24. Morasso MC, Molero J, Vinocur P, Acosta L, Paccussi N, Raselli S, et al. Iron and vitamin A deficiencies and prevalence of anemia in boys and girls between 6 to 2 months of age in Chaco, Argentina. Arch Lationam Nutr 2003; 53:21-7.

25. Tatala S, Svanberg U, Mduma B. Low dietary iron availability is a major cause of anemia: a nutrition survey in the Lindi of Tanzania. Am J Clin Nutr 1998; 68:171-8.

26. Wharf SG, Fox TE, Fairweather-Tait SJ, Cook JD. Factors preventing iron stores in infants 4-18 months of age. Eur J Clin Nutr 1997; 51:504-9.

27. Thane CW, Bates CJ, Prentice A. Risk factors for low iron intake and poor iron status in a national sample of British young people aged 4-18 years. Public Health Nutr 2003; 6:485-96.

28. Domellof M, Lonnerdal B, Dewey KG, Cohen RJ, Rivera LL, Hernell O. Sex differences in iron status during infancy. Pediatrics 2002; 110:545-52.

29. Lima ACVMS, Lira PIC, Romani SAM, Eickmann SH, Piscoya MD, Lima MC. Fatores determinantes dos níveis de hemoglobina em crianças aos 12 meses de vida na Zona da Mata Meridional de Pernambuco. Rev Bras Saúde Matern Infant 2004; 4:35-43.

30. Muller O, Krawinkel M. Malnutrition and health in developing countries. CMAJ 2005; 173:279-86.

31. Osorio MM. Fatores determinantes da anemia em crianças. J Pediatr (Rio J.) 2002; 78:269-78.

32. Agho KE, Dibley MJ, D’Este CD, Gibberd R. Factors associated with haemoglobin concentration among Timor-Leste children aged 6-59 months. J Health Popul Nutr 2008; 26:200-9.

33. Levy A, Fraser D, Shirley DR, Dagan R, Deckelbaum RJ, Coles C, et al. Anemia as a risk factor for infectious diseases in infants and toddlers: results from a prospective study. Eur J Epidemiol 2005; 20:277-84.

34. Beresford CH, Neale RJ, Brooke OG. Iron absorption and pyrexia. Lancet 1971; i:568-72.

35. Walter T, Olivares M, Pizarro F, Munoz C. Iron, anemia, and infection. Nutr Rev 1997; 55:111-24.

36. Rey L. Um século de experiência no controle da ancilostomíase. Rev Soc Bras Med Trop 2001; 34:61-7.

37. Ferreira MU, Ferreira CS, Monteiro CA. Tendência secular das parasitoses intestinais na infância na cidade de São Paulo (1984-1996). Rev Saúde Pública 2000; 34:73-82.

38. Benicio MHDA, Monteiro CA. Tendência secular da doença diarréica na infância na cidade de São Paulo (1984-1996). Rev Saúde Pública 2000; 34:83-90.

Recebido em 14/Jun/2010

Versão final reapresentada em 09/Ago/2010 Aprovado em 10/Set/2010 